\title{
On the role of enthalpic and entropic contributions to the conforma- tional free energy landscape of MIL-101(Cr) structural building units
}

\author{
Loukas Kollias $^{1}$, David C. Cantu ${ }^{2}$, Vassiliki-Alexandra Glezakou ${ }^{3 *}$, Roger Rousseau ${ }^{3}$, Matteo Salvalaglio ${ }^{1^{*}}$ \\ ${ }^{I}$ Thomas Young Centre and Department of Chemical Engineering, University College London, London WC1E 7JE, United Kingdom; \\ ${ }^{2}$ Chemical and Materials Engineering Department, University of Nevada, Reno, Reno, NV, 89557, United States; \\ ${ }^{3}$ Basic and Applied Molecular Foundations, Pacific Northwest National Laboratory, Richland, WA, 99352 United States \\ KEYWORDS: Molecular Dynamics, Metal-Organic Frameworks, Entropy
}

\begin{abstract}
The thermostructural behavior of metal-organic framework (MOF) precursors is responsible for regulating the introduction of MOF structure defects during synthesis. In this paper, we evaluate factors affecting the flexibility of MIL-101(Cr) halfsecondary building units (half-SBUs) in solution using enhanced sampling methods. In particular, we calculate entropic and enthalpic contributions to the conformational free energy landscape of isolated MIL-101(Cr) half-SBUs, in water, in the presence and absence of ionic species (Na+ and F-), and in N, N-dimethylformamide (DMF). We find that the interplay between enthalpy and entropy determines the most probable conformational state for half-SBUs. Furthermore, we investigate the role of enthalpy and entropy in the conformational rearrangement of an SBU in water, noting that entropic contributions are essential to stabilize configurations that depart from those coherent with the MIL-101(Cr) crystal structure. Our analysis highlights the importance of explicitly considering entropic effects on the configurational ensembles of MOF building units and highlights the significant impact they have on the relative stability of crystal-like and non-crystal-like conformers.
\end{abstract}

\section{INTRODUCTION}

Elucidating the nature of enthalpic and entropic contributions is of significant importance for several processes in organometallic chemistry ${ }^{1-3}$ such as in peptide - metal nanoparticle ${ }^{3}$ systems. Surface stability ${ }^{4}$, solute aggregation ${ }^{5}$, supramolecular encapsulation ${ }^{6}$ and preferential adsorption of compounds ${ }^{7}$ are a few examples of entropically driven processes. Also, entropy - enthalpy compensation phenomena in small solvated solutes, especially proteins, are of particular scientific interest to thoroughly understand the functioning mechanisms of molecules in solution ${ }^{1,6,8-12}$.

Flexibility is associated with configurational entropy and this can determine the structure and functionality of a molecule ${ }^{13}$. Metal-organic frameworks (MOFs) are built out of flexible building units. A rich conformational ensemble unveiled during the association-dissociation of secondary building units (SBUs) ${ }^{14}$ highlights the importance of understanding the origin of the contributions to the free energy landscape of half-SBUs. We recently proposed that the early stages of MOF nucleation are defined by the assembly of half-SBUs into SBU-containing MOF precursors ${ }^{14-15}$. However, the drivers of SBU and halfSBU flexibility are yet to be understood from a thermodynamics perspective.

For instance, the propensity of healing defects in the crystal lattice can be linked with the population of different isomers as well as the guest molecules present during the nucleation stages ${ }^{14}$. Therefore, it is essential to trace back to the origins of this selectivity and understand how different isomers function in the presence of guest molecules. A regulation of the population of defects, which is highly desirable in MOF synthesis ${ }^{16-19}$, can thus be rationalized and achieved.
Assessing the entropic and enthalpic profiles of half-SBUs can prove essential to manipulate the thermostructural behavior of the MOFs, which are considerably less thermally stable than zeolites $^{20}$. Nevertheless, MOFs are significantly more porous. Therefore, analyzing their thermostructural behavior can provide insights into improving their robustness for a plethora of applications. Mechanical flexibility ${ }^{21}$ and thermal expansion ${ }^{22}$ of MOFs have been investigated in previous works. Nevertheless, the enthalpic and entropic drivers to the assembly free energy of building units were not assessed. Computational ${ }^{20,23-24}$ and experimental ${ }^{25}$ studies on enthalpic and entropic contributions to the adsorption free energy have been frequently carried out for $\mathrm{MOFs}^{20,23,25}$ and metal clusters ${ }^{24}$ with a focus on applications. In the field of MOF synthesis, there is a growing effort to improve the mechanical stability of MOFs by taking advantage of novel techniques ${ }^{26-28}$. However, understanding of the entropic and enthalpic contributions to the free energy of halfSBU flexibility is an underexplored area, which has the potential to devise strategies on improving MOFs' intrinsic characteristics during synthesis.

In this work, we evaluate entropic-enthalpic contributions to the free energy profile associated with structural fluctuations in half-SBUs and SBUs. This analysis allows for an evaluation of the conformational freedom that different molecules experience in solution. This, in turn, leads to a detailed understanding of their flexibility and provides further insight into the nature of the configurational ensembles likely to be visited during selfassembly and nucleation. In addition to this analysis, we calculate the inertia tensor for each isomer, of the MIL-101(Cr) half$\mathrm{SBU}^{14-15}$, as well as its solvation behavior in different environ- 
ments. These complementary pieces of information provide detailed insight into the molecular behavior of MOF building units which drives self-assembly and self-healing during synthesis.

\section{METHODS}

\section{Simulation setup}

Molecular dynamics (MD) simulations were performed in explicit solvent. The leapfrog integrator was used to propagate dynamics of the system with a time step of 2 fs. The LINCS ${ }^{29}$ algorithm preserved the distances of bonds involving hydrogen atoms. The cutoff for non-bonded interactions is $10 \AA$. Long range electrostatics were treated using the Particle-Mesh Ewald $(\mathrm{PME})^{30}$ scheme. The velocity rescaling thermostat ${ }^{31}$ and the Berendsen barostat ${ }^{32}$ preserved the temperature and pressure at $298 \mathrm{~K}$ and 1 bar respectively. The system of isolated half-SBUs (MLA, MLB and MLC) ${ }^{14-15}$ in solution, which consists of 6500 atoms, was simulated in a cubic box of edge $4.0 \mathrm{~nm}$ with periodic boundary conditions in three dimensions. The system of an SBU in solution has 12000 atoms in a cubic cell with edge equal to $5.0 \mathrm{~nm}$. Solvent has been treated explicitly with the TIP3P model $^{33}$ and ions with the OPLS-AA force field ${ }^{34}$. The force filed for the half-SBU species is discussed in detail in a previous work $^{14-15}$. Simulations were carried out using GROMACS $2018^{35}$ and PLUMED 2.5 $5^{36}$. Chemical structures were visualized using $\mathrm{VMD}^{37}$. All the data and PLUMED input files required to reproduce the results reported in this paper are available on PLUMED-NEST (www.plumed-nest.org), the public repository of the PLUMED consortium ${ }^{38}$, as plumID:19.073.

Well-tempered metadynamics (WTmetaD) ${ }^{39}$ is employed to enhance sampling and construct free energy surfaces along chemically intuitive collective coordinates. In this context, we construct a bias potential as a function of the mass-weighted radius of gyration $\left(R_{\text {gyr }}\right)$ to assess the flexibility of the MIL-101(Cr) half-SBUs ${ }^{15}$. Gaussians of width $0.01 \mathrm{~nm}$ and initial height $0.5975 \mathrm{kcal} / \mathrm{mol}$ were deposited every $1 \mathrm{ps}$ with a bias factor of 10 in absence of ions, and a bias factor of 50 in presence of ions. The total simulation time in water in absence and presence of ions $(0.25 \mathrm{M})$, and DMF was $0.5 \mu \mathrm{s}$.

Furthermore, a simulation studying the rearrangement of an SBU (MLA - MLA or AA for simplicity) in solution was performed through biasing the coordination numbers greater than 0.5 with a coordination sphere of radius $0.25 \mathrm{~nm}$, and the total potential energy of the system for $1.5 \mu \mathrm{s}$. Gaussians of width 0.01 and $47.8 \mathrm{kcal} / \mathrm{mol}$ for coordination number and potential energy were deposited every 1 ps with initial height and bias factor equal to $0.598 \mathrm{kcal} / \mathrm{mol}$ and 100 respectively. A rational switching function ${ }^{36}$ with parameters $\left(d_{0}=0, n=2, m=4\right)$ was used in order to calculate the set of coordination numbers. At last, a harmonic restraining potential $\left(V_{r}\right)$ acted on the lowest component of the set of all distances between any chromium and any terminal carboxylic carbon atoms. The potential, characterized by a force constant of $35.85 \mathrm{kcal} / \mathrm{mol}$ was active when the minimum distance exceeded $3.5 \AA$.

\section{Enthalpic and entropic contributions to the conformational} free energy landscape.

As a result of WTmetaD calculations, a free energy surface is the direct output, as a projection of the free energy of the system $G$ in the space of collective variables, denoted with $\boldsymbol{s}^{39}$. The
Gibbs free energy can be expressed as the sum of its enthalpic and entropic contributions, i.e. $\Delta \mathrm{G}(\mathbf{s})=\Delta \mathrm{H}(\mathbf{s})-\mathrm{T} \cdot \Delta \mathrm{S}(\mathbf{s})$, where $\mathrm{G}(\mathbf{s})$ is the free energy, $\mathrm{H}(\mathbf{s})$ the enthalpy, and $\mathrm{S}(\mathbf{s})$ the entropy, and $\Delta$ represents the difference with respect to an arbitrarily chosen reference state in $\mathbf{s}^{40}$.

To systematically decompose the free energy surface $\Delta \mathrm{G}(\mathbf{s})$ we employ the method discussed in Ref. ${ }^{40}$, which is based on mapping the enthalpic component of the free energy in $\mathbf{s}$ and obtaining the entropic contribution to free energy by difference.

In the case of conformational transitions in solution, associated to negligible variations in the excluded volume, the $P \Delta \mathrm{V}(\mathbf{s})$ contribution to enthalpy (where $P$ is the pressure and $V(\mathbf{s})$ the volume mapped in $\mathrm{CV}$ space) is constant. Hence $\Delta \mathrm{H}(\mathbf{s})$ reduces to the internal energy $\Delta \mathrm{U}(\mathbf{s})$. Furthermore, at constant $\mathrm{T}$, the kinetic energy does not depend on $\mathbf{s}$, and therefore the internal energy contribution $\Delta \mathrm{U}(\mathbf{s})$ further reduces to the potential energy of the system $\Delta E_{P}(\mathbf{s})^{40}$.

Since in this work we are analyzing conformational transitions in half-SBUs or SBUs embedded in a solvent, we shall highlight that the potential energy of the system is dominated by the potential energy of the solvent. To limit the statistical uncertainty in the decomposition of the free energy surface $\Delta \mathrm{G}(\mathbf{s})$, we shall therefore further decompose the contributions to $\Delta E_{P}(\mathbf{s})$ as follows:

$$
\Delta E_{P}(\mathbf{s})=\Delta E_{P}^{\text {solute }}(\mathbf{s})+\Delta E_{P}^{\text {solute-solvent }}(\mathbf{s})+\Delta E_{P}^{\text {solvent }}(\mathbf{s})
$$

Where: $E_{P}^{\text {solute }}(\mathbf{s})$, and $E_{P}^{\text {solvent }}(\mathbf{s})$ represent the potential energy of the solute, including all the bonded terms (bond, angles, proper dihedrals and Ryckaert-Bellemans dihedrals ${ }^{41}$ ) of solute and solvent respectively; plus the non-bonded, short-range terms for solute-solute and solvent-solvent interactions.

The term $E_{P}^{\text {solute }}(\mathbf{s})$ accounts for the non-bonded, short-range potential energy terms associated to solute-solvent interactions. We shall note that the term $E_{P}^{\text {solvent }}(\mathbf{s})$ is independent from the conformation of the solute mapped on $\mathbf{s}$, hence its contribution to $\Delta E_{P}(\mathbf{s})$ is negligible.

In order to map the ensemble averages of the term $\Delta E_{P}^{\text {solute }}(\mathbf{s})+\Delta E_{P}^{\text {solute-solvent }}(\mathbf{s})$ in $\mathbf{s}$, as discussed in Gimondi et al. ${ }^{40}$ we use the reweighting method of Tiwary and Parrinello $^{42}$

\section{Solvation shell}

In order to investigate the role of the solvation shell on the enthalpy and entropy of rearrangement of half-SBUs, we calculate contributions to the potential energy by changing our convention for the molecules considered in the calculation of the term $\Delta E_{P}^{\text {solute-solvent }}(\mathbf{s})$.

In particular we now explicitly distinguish between solvent molecules found within a certain radius from the solute, constituting the solvation shell interacting with the SBUs. By considering solvation shells of increasing size (from 2.5 to $20 \AA$ ), and monitoring the convergence of the potential energy contribution as a function of the solvation shell size, we quantify the impact of the SBUs conformational re-arrangement on the surrounding solvent environment, and assess solvation shell impact on the conformational entropy.

\section{Effect of ions and solvent}

The role of ions and the changes in the free energy and the enthalpy - entropy profiles are investigated. Ions $\left(\mathrm{Na}^{+}, \mathrm{F}^{-}\right)$are 
added at a concentration of $0.25 \mathrm{M}$, with respect to the simulation box, in order to evaluate half-SBU flexibility in excess of ionic species. We complement the interactions between the solute and the solvent with those between the ionic species and the solute. Furthermore, a different solvent can significantly af- fect the energetics of conformational transformations in the solute. This is due to entropic and enthalpic contributions being affected by the nature of solvation ${ }^{6}$. To assess this effect on MIL-101(Cr) SBUs, in addition to water we investigate $\mathrm{N}, \mathrm{N}-$ dimethylformamide (DMF). The OPLS forcefield parameter for DMF were obtained from the virtualchemistry.org database $\mathrm{e}^{43-44}$.
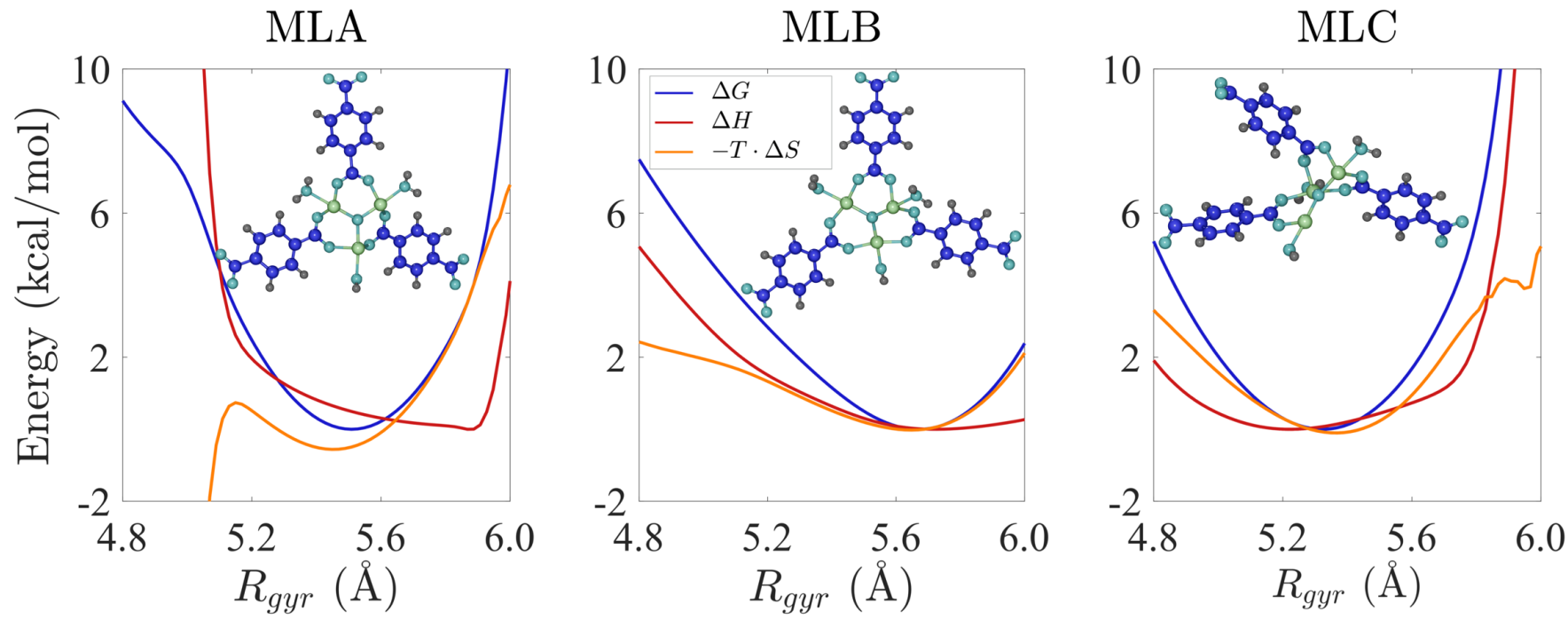

Figure 1. Energetic landscape of MLA, MLB and MLC in water as a function of the $R_{g y r}$. In blue we report the free energy profile, red: enthalpic contribution, orange: entropic contribution profile. Molecular configurations shown correspond to structures representative of the free energy minima in the respective profiles. In all cases $\mathrm{T}=298 \mathrm{~K}$. Color code: $\mathrm{Cr}-$ lime, $\mathrm{C}-$ blue, $\mathrm{O}-$ cyan and $\mathrm{H}-\mathrm{gray}$.

\section{RESULTS}

We investigate the driving forces to the conformational complexity of the half-SBUs of MIL-101(Cr $)^{45}$ by exploring the energetic profile of $\mathrm{R}_{\mathrm{gyr}}$. We carry out this analysis for all three half-SBU isomers identified as key in the early stages of MIL$101(\mathrm{Cr})$ self-assembly ${ }^{15}$. The structural differences between these conformers render distinct free energy profiles projected on $\mathrm{R}_{\mathrm{gyr}}$. In order to thoroughly understand the origins of these discrepancies, we calculate the entropic and enthalpic contributions to the free energy for each structure.

A first observation is that MLA has considerably different free energy and entropic contribution minima, unlike MLB and MLC. In MLA, entropy favors compact configurations, while enthalpy drives the system to larger values of $R_{\text {gyr }}$. MLB presents similar values of $\mathrm{R}_{\mathrm{gyr}}$ for the strongest enthalpic and entropic contributions to the free energy, corresponding to the largest value of $\mathrm{R}_{\mathrm{gyr}}$ among the three half-SBUs. MLC presents the minimum enthalpic contribution at a low value of $\mathrm{R}_{\mathrm{gyr}}$; hence rendering more compact configurations enthalpically favored. Another straightforward observation is that the minima for the enthalpic and entropic contributions correspond to similar values for $R_{\mathrm{gyr}}$ as in MLB, while for MLA the respective $\mathrm{R}_{\mathrm{gyr}}$ values differ by more than $1 \AA$.

To summarize the analysis of the energetic landscape for halfSBUs in water, the free energy of MLA is dominated by strongly contrasting enthalpic and entropic effects. The former favors "open" structures with stretched arms, while the latter favors more compact configurations. In MLB and MLC, enthalpy and entropy contribute in a similar manner to the free energy profile, with their minima exhibiting close values of $R_{\mathrm{gyr}}$. The resulting energy profiles are reported in Fig. 1.

\section{Effect of solvation shell}

The effect of the solvation shell on the entropic - enthalpic contribution profiles is investigated. Our motivation stems from the need of assessing the interplay between entropy and enthalpy in relation to solvation. For instance, strong enthalpic interactions between the solute and the solvent molecules, within a solvation shell, can induce significant changes in the entropy of these molecules against those far away from the solute ${ }^{9}$. Recently, Schauperl et al. ${ }^{46}$ showed that the water model choice can only result in minor differences in the entropy and enthalpy of solvation. An exception where the TIP $3 \mathrm{P}^{33}$ model shows lower degree of order for molecules around the solute refers to amino acids, which are not of interest in this work.

We consider six solvation shell sizes: $2.5,5,7.5,10,15$ and 20 $\AA$. The resulting profiles for three cutoff distances are provided in Fig. 2. We note that entropy/enthalpy breakdown converges for shell sizes larger than $5 \AA$. Calculating the differences in the enthalpy contribution at different shell sizes we conclude that a solvation shell of $7.5 \AA$ is sufficient to capture solute-solvent interactions. For instance, accounting for a solvation shell of size $7.5 \AA$ produces a maximum difference with respect to the enthalpy profile obtained including all the solvent molecules of the order of $k_{B} T$. Such a difference becomes negligible for solvation shell sizes larger than $10 \AA$. 


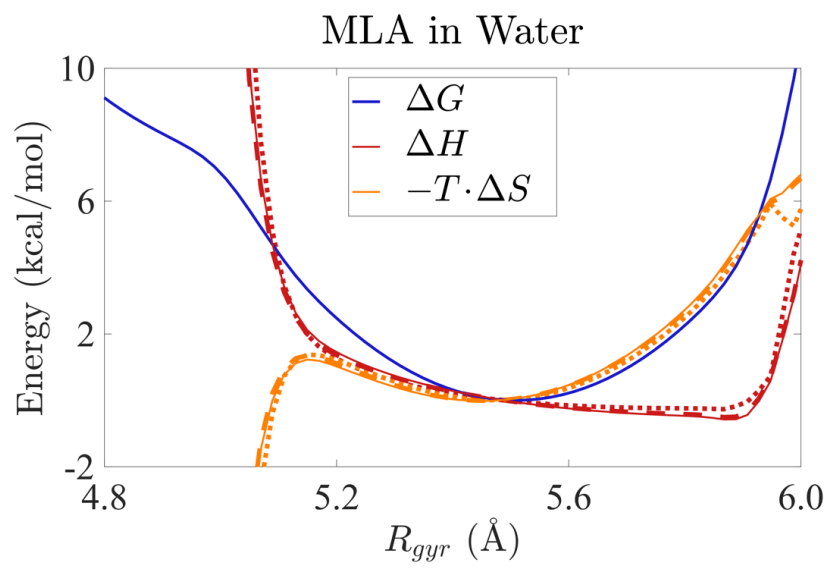

Figure 2. Energetic landscape of MLA in water as a function of $R_{\text {gyr }}$. Contributions of an increasing solvation shell are provided. Blue: free energy, red: enthalpic contribution, orange: entropic contribution profile. Line types distinguish between solvation shell sizes, dot: $2.5 \AA$, bold dash: $5 \AA$, solid: $10 \AA$.

This observation indicates the spatial impact of solute reorganization on the energetics of the system, which extends to the solution environment, including solvent molecules within 7.5 $10 \AA$. We note that the free energy profile is inherently associated to the configurational space of the entire simulation box, and it is not affected by changes in the size of the solvation shell. An analogous analysis has been carried out for MLA in aqueous solution, in the presence of ions, and in DMF. Very similar trends are observed in water in absence or presence of ions. As in pure water the solute-solvent interactions are fully captured

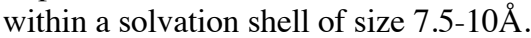

In DMF the trend changes as a cut-off of $7.5 \AA$ is needed to qualitatively represent the enthalpic contributions. A $10 \AA$ cutoff is thus the minimum solvation shell size required for this purpose. The enthalpy and entropy profiles computed in the presence of ions and in DMF as a function of solvation shell size are reported in the Supporting Information (SI), section IV.

In order to understand the effect of solvation on the enthalpic and entropic contributions to the free energy profiles, the radial and cumulative distribution functions are calculated between the central oxygen of the metal center of each half-SBU $\left(\mathrm{O}_{\mathrm{u}}\right.$ MLA) and a characteristic atom of each solvent (the oxygen for water and the nitrogen for DMF). Radial distribution functions are reported in SI, figure S4. In water, the first solvation shell is located $3 \AA$ away from the central oxygen of MLA in water, in absence and presence of ions, and it contains 3 solvent molecules. The second solvation shell displays a bimodal structure, with the first peak located at $5.2 \AA$. At $5.7 \AA$, water displays a secondary peak in the second solvation shell, while in the presence of ions the second solvation shell is represented by a mono-modal peak centered at 5.7 $\AA$. The third solvation shell in water is located between 7.5-8 $\AA$ in water, both in the presence or absence of counterions. This analysis highlights that the impact on the energetics of the system, induced by the conformational rearrangement of MLA extend up to the fourth coordination shell, involving up to 46 water molecules.
In DMF, the first solvation shell is centered around $5.2 \AA$ with the second solvation shell located at $8 \AA$. This indicates that in DMF the solute-solvent interplay in the definition of the enthalpic and entropic contributions to the conformational free energy landscape only extend up to the second solvation shell, involving approximately 11 molecules.

\section{Addition of ions}

The addition of ions (at a concentration of $0.25 \mathrm{M}$ ) can significantly alter the energetics of the system due to their strong interactions with partially charged atoms of the half-SBU. We analyze contributions to the MLA free energy profile as this presents the strongest electrostatic interactions. The introduction of ionic species results in additional electrostatic and van der Waals contributions to the potential energy with a net effect in favor of electrostatics as the total potential energy is shifted to more negative values compared to the simulations in absence of ions. The free energy profile is considerably less steep than in absence of ions; hence there is a much larger number of configurations which supply similar entropic contributions to the free energy. Furthermore, the $\Delta \mathrm{H}$ profile presents a minimum that is close to the free energy minimum. As a result, a maximum entropic contribution is observed in this area. This analysis shows that in presence of ions the free energy is dominated by enthalpy which tend to favor slightly more compact configurations than those corresponding to the free energy minimum.

Significant contributions to the potential energy stem from the interactions of fluoride anions with chromium atoms of the halfSBU metal center which restrict free movement of the linkers. In further detail, a rapid shift to a stronger and relatively constant contribution is observed in the trajectory of the electrostatic interaction, when the three chromium atoms are coordinated with two fluoride anions.

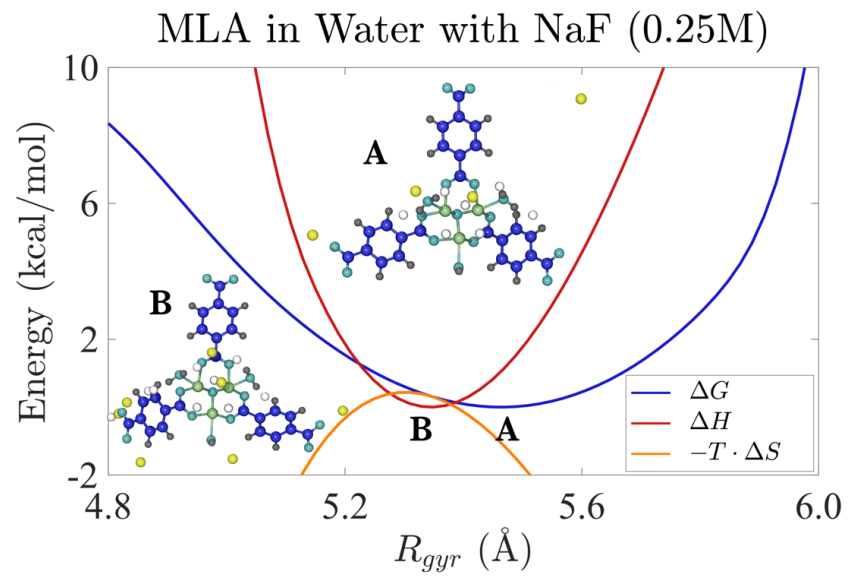

Figure 3. Energetic landscape of MLA in water with $0.25 \mathrm{M} \mathrm{NaF}$ as a function of $R_{g y r}$. Blue: free energy, red: enthalpic contribution, orange: entropic contribution profile. Structure A corresponds to the free energy minimum and B to the enthalpic contribution minimum. The color code is consistent with Fig. 1.

This highlights that ions entropically alter the energetic landscape of the half-SBU flexibility by increasing the set of accessible configurations at a certain value of $\mathrm{R}_{\mathrm{gyr}}$; hence increasing the level of degeneracy in the space of $R_{\mathrm{gyr}}$. The latter results in a much wider free energy well compared to the corresponding 
profiles in pure water. At last, we observe that $\mathrm{Cr}$ in the metal center tend to ineteract with three $\mathrm{F}^{-}$through strong electrostatic interactions.

The resulting energetic profile for MLA is available in Fig. 3. Energetic profiles for MLB, MLC in water in presence of ions are available in the SI, section III.

\section{Effect of solvent}

The effect of using DMF instead of water becomes apparent for MLA when the potential energy is projected on collective variable space. Unimodal enthalpy and entropy profiles emerge in contrast with water, where two distinct states appear. Also, both provide similar contributions to free energy as it can be seen in Fig. 4. Nevertheless, the minimum for the entropic contribution is relevant to the one in water for a value of $R_{\text {gyr }}$ less than $4.8 \AA$, while the enthalpic contribution shows a minimum for values close to $5.7 \AA$.

This observation leads to the conclusion that in DMF the free energy minimum is very close to the one obtained in water, but the underlying entropic and enthalpic contributions are considerably different. This means that the configurations corresponding to low values of $\mathrm{R}_{\mathrm{gyr}}$; and hence corresponding to more compact structures, do not belong to very high potential energy regions, as in water. In other words, DMF does not favor a plethora of entropically accessible states for very compact structures due to a flat profile of entropic contribution for $\mathrm{R}_{\mathrm{gyr}}$ smaller than the value corresponding to the free energy minimum. This bulkier solvent hinders the flexibility of the half-SBU when in compact form; thus, resulting to rigid configurations at low values of $R_{\mathrm{gyr}}$. This rigidity can be linked with the study of the more stable forms of SBUs in DMF as discussed in another work ${ }^{14}$. At last, energetic profiles for MLB and MLC in DMF are available in the SI, section III.

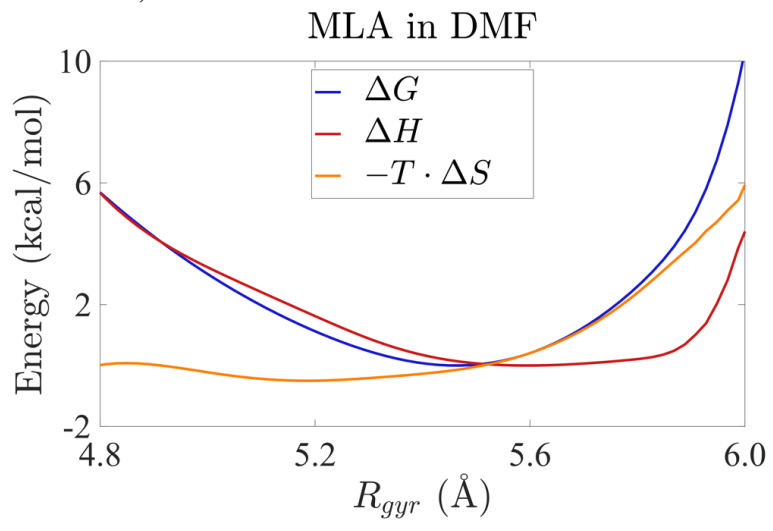

Figure 4. Energetic landscape of MLA in DMF as a function of $R_{\text {gyr }}$. Blue: free energy, red: enthalpic contribution, orange: entropic contribution profile.

\section{Isomer symmetry}

Another important aspect that can provide significant insight into the conformational flexibility of different isomers is a measure of symmetry along principal axes of inertia. The moments of inertia corresponding to rotational movement around principal axes are calculated and mapped on collective variable space. We calculate the major, middle and minor axes of inertia and the corresponding moments. We observe that the major axis is similar for the three isomers. The middle and minor axes are appreciably different for MLA compared with the other two. Nevertheless, the middle axis for MLA is similar to the minor axis for MLB and MLC.

The moment of inertia corresponding to the major axis has the highest favourable value for MLA and the lowest for MLC. Also, MLA and MLB present a narrower range of values compared with MLC where the moment of inertia has a very wide distribution. The moment of inertia corresponding to the middle axis has the lowest favorable value for MLA and the highest for MLC. Again, MLA and MLB present a narrower range when compared with MLC. The moment of inertia corresponding to the minor axis presents a narrow distribution and the lowest favorable value for MLC. For MLB, the opposite occurs as it has the highest favorable value and the wider distribution. In addition to water, we assess the probability density of moments of inertia in DMF. Moments that correspond to the major and middle axes show very similar densities, while rotation around the minor axis renders the total moment of inertia for MLA lower than in water and close to MLC, corresponding to a more compact structure that allows the molecule to possess smaller rotational inertia. This analysis offers a potential explanation to the observation that MLA results in more compact SBUs in $\mathrm{DMF}^{14}$. Overall, MLA resists rotational movement around its major axis, while it is more probable to rotate around its middle axis. Also, it has appreciably narrower distributions for its moments of inertia around the major and middle axis than MLB and MLC. MLB has a relatively low tendency to rotate around its major and middle axis and has a wide distribution of moments of inertia corresponding to its minor axis. MLC is more likely to rotate around its major axis, while it presents the widest distributions for its moments of inertia corresponding to its major and middle axis. At last, the trend for the total moment of inertia for rotational movement around any axis has a trend that is very similar to $R_{\text {gyr }}$ for all isomers. This analysis is available in the SI, section I.

\section{Study of the rearrangement of an SBU}

At last, we depart from the assessment of isolated half-SBUs in solution in order to evaluate entropic and enthalpic contributions to the free energy landscape of a half-SBU couple forming an SBU in solution. We start the simulations from a crystal-like SBU and sample the configuration space of different arrangements based on the coordination between terminal carboxylic oxygen atoms of one half-SBU with the chromium atoms at the metal center of the adjacent half-SBU. In the collective variable space, a value of 1.6 corresponds to 2 chromium atoms as in the crystal lattice, labelled $\mathrm{C}$ for crystal-like. A coordination number of 2.5 is representative of the 3 chromium - oxygen interactions, labelled $\mathrm{T}$ (for triple chromium oxygen interaction). A coordination number of 3.3 to 4 chromium - terminal carboxylic oxygen interactions, labelled Q (indicating a quadruple chromium-oxygen interaction). The energetic profiles of the SBU re-arrangement show that $\mathrm{C}$ is enthalpically dominated, while $\mathrm{Q}$ is entropically dominated. Since, Q is the most stable structure from a free energy standpoint, we conclude that the free energy landscape of the re-arrangement of an SBU is entropically dominated as seen in Fig. 5. Since, the potential energy profile after a simulation of $1.5 \mu \mathrm{s}$; we performed a cubic spline interpolation in order to show the enthalpic and entropic contributions to the SBU rearrangement. Energetic profiles are 
reweighted in order to negate the effect of the bias due to both WTmetaD ${ }^{39}$ and $V_{r}$. In the sake of completeness, the actual profiles are also shown in Fig. 5. Finally, we have calculated the average error on the free energy projected on either the coordination number or the enthalpy. This is based on block analysis, partitioning the trajectory in blocks of sizes ranging from 10 to
$100 \mathrm{~ns}$. Thereafter, we report that the error is below $0.1 \mathrm{kcal} / \mathrm{mol}$ for the coordination number and below $\mathrm{k}_{\mathrm{B}} \mathrm{T}$ for enthalpy.

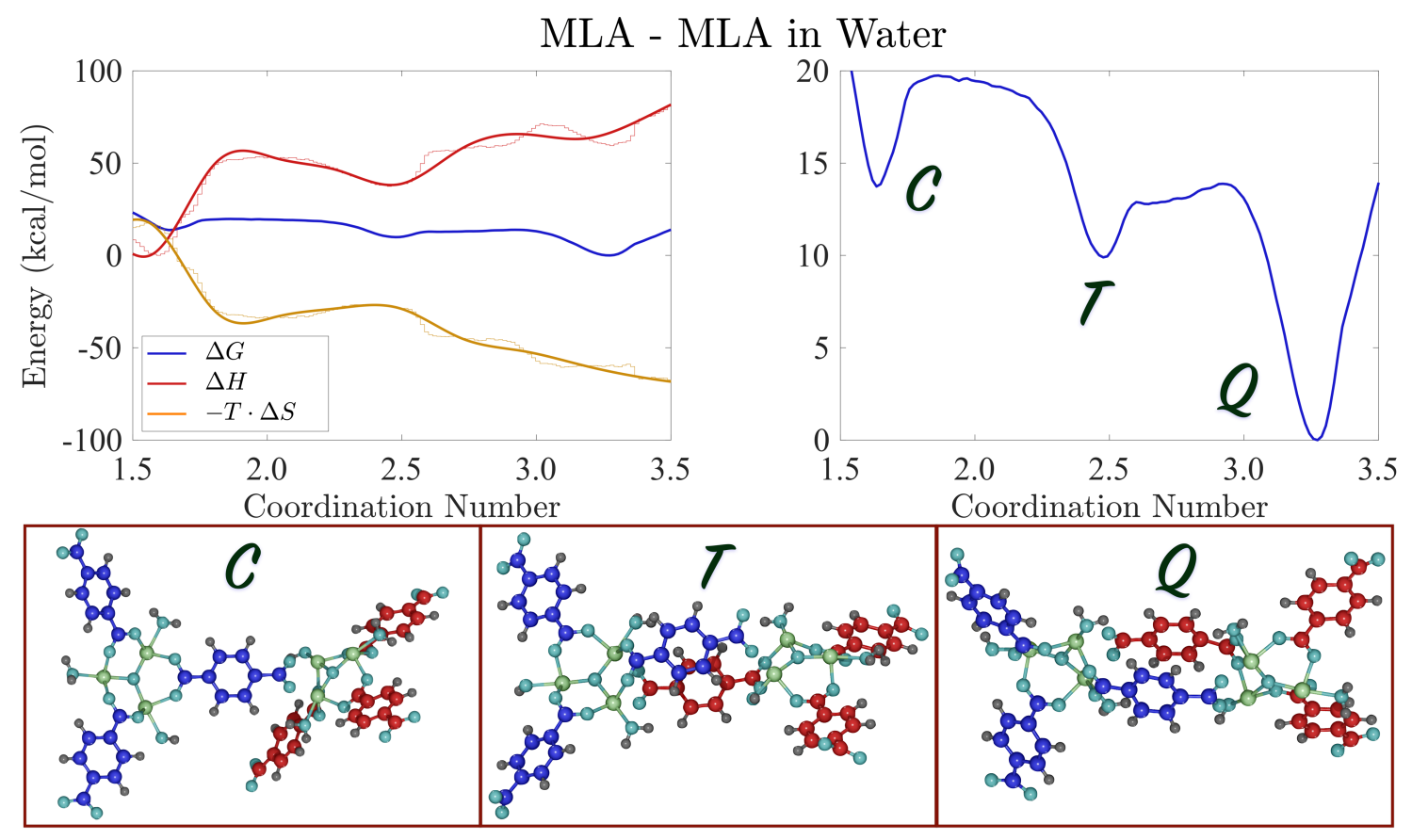

Figure 5. Energetic landscape of rearrangement of an AA SBU in water with respect to the coordination number between terminal carboxylic oxygen and adjacent chromium atoms. Left panel: Blue: free energy, red: enthalpic contribution, orange: entropic contribution profile. Bold line plots for enthalpy and entropy represent a cubic spline interpolation of the actual profiles (thin line plots). Right panel: free energy profile in detail. Configurations corresponding to the $\mathrm{C}, \mathrm{T}$ and $\mathrm{Q}$ states are shown. The color code is consistent with Fig. 1.

\section{CONCLUSIONS}

In this work, we investigate the entropic and enthalpic contributions to the free energy landscape of MOF building units. This enhances our understanding of how the thermostructural properties of MOF precursors can be fine-tuned. The behavior of MIL-101(Cr) half-SBU isomers ${ }^{15}$ is evaluated in different environments, including water, in absence and presence of ions, and $\mathrm{DMF}$, in order to examine the drivers that can render them sources of either crystal-like units or defects ${ }^{14}$. In this effort, we use the mass-weighted radius of gyration $\left(R_{\text {gyr }}\right)$ as a measure of isomer-specific molecular flexibility.

In water, MLB and MLC present free energy profiles with similar enthalpic and entropic contributions in the space of $\mathrm{R}_{\mathrm{gyr}}$. The free energy minima correspond to $\mathrm{R}_{\mathrm{gyr}}$ values between the minima for entropic and enthalpic contributions. In MLA, the entropic contribution presents a minimum for very low $\mathrm{R}_{\mathrm{gyr}}$, while the enthalpic contribution minimum corresponds to a configuration with high $R_{\text {gyr }}$ value. The presence of ions increases the number of accessible states in the space of $R_{\mathrm{gyr}}$ sampled during the simulation. Solvent effects have a considerable impact as the enthalpic and entropic contribution minima for MLA are close and the compact configuration that correspond to the entropic contribution minimum in pure water is highly unfavorable in bulkier solvents such as DMF.
At last, we study the rearrangement of a SBU in water, a process that has been identified as relevant in a previous work ${ }^{14}$. Our analysis shows that the crystal-like configuration of the SBU (C) is enthalpically favored, while SBUs configurations displaying multiple concurrent linker-metal center interactions $(\mathrm{T}$, Q) are entropically favored. This allows to identify the configurational entropy contributions as the main driver for the formation of SBUs that depart from the crystal-like configuration and are likely to result in defective materials. Our findings highlight how static potential energy calculations provide only a partial and perhaps insufficient description of the configurational landscape of MOF building units.

\section{ASSOCIATED CONTENT}

\section{Supporting Information}

The Supporting Information is available free of charge on the ACS Publications website.

Supplementary information (SI) linked with the main article (PDF)

\section{AUTHOR INFORMATION}

\section{Corresponding Author}

*vanda.glezakou@pnnl.gov

*m.salvalaglio@ucl.ac.uk 


\section{Notes}

The authors declare no competing financial interest.

\section{ACKNOWLEDGMENT}

The work described in this publication was performed by Pacific Northwest National Laboratory (PNNL), which is operated by Battelle for the United States Department of Energy (DOE) under Contract DE-AC05-76RL0180. V.-A. G. and R. R. acknowledge support from DOE Office of Basic Energy Sciences - Chemical, Geological and Biological Sciences Division. L. K. was partially supported by a Laboratory Directed Research and Development grant for PNNL. D.C.C. acknowledges support from Research and Innovation at the University of Nevada, Reno. The authors acknowledge the use of the UCL Myriad High Performance Computing Facility (Myriad@UCL), and associated support services, in the completion of this work. We are grateful to the UK Materials and Molecular Modelling Hub for computational resources, which is partially funded by EPSRC (EP/P020194/1). This research used resources of the National Energy Research Scientific Computing Center, a DOE Office of Science of the U.S. Department of Energy under Contract No. DE-SUPPLEMENTARY MATERIAL AC02$05 \mathrm{CH} 11231$.

\section{REFERENCES}

1. Choudhury, N.; Pettitt, B. M., Enthalpy-entropy contributions to the potential of mean force of nanoscopic hydrophobic solutes. J Phys Chem B 2006, 110 (16), 8459-63.

2. van der Vegt, N. F. A.; van Gunsteren, W. F., Entropic Contributions in Cosolvent Binding to Hydrophobic Solutes in Water. The Journal of Physical Chemistry B 2004, 108 (3), 1056-1064.

3. Tang, Z.; Palafox-Hernandez, J. P.; Law, W.-C.; Hughes, Z. E.; Swihart, M. T.; Prasad, P. N.; Knecht, M. R.; Walsh, T. R., Biomolecular Recognition Principles for Bionanocombinatorics: An Integrated Approach To Elucidate Enthalpic and Entropic Factors. ACS Nano 2013, 7 (11), 9632-9646.

4. Amirjalayer, S.; Tafipolsky, M.; Schmid, R., Surface Termination of the Metal-Organic Framework HKUST-1: A Theoretical Investigation. The Journal of Physical Chemistry Letters 2014, 5 (18), 3206-3210.

5. Mondal, J.; Yethiraj, A., Driving Force for the Association of Amphiphilic Molecules. The Journal of Physical Chemistry Letters 2011, 2 (19), 2391-2395.

6. Leung, D. H.; Bergman, R. G.; Raymond, K. N., Enthalpy-Entropy Compensation Reveals Solvent Reorganization as a Driving Force for Supramolecular Encapsulation in Water. Journal of the American Chemical Society 2008, 130 (9), 2798-2805.

7. Van de Voorde, B.; Boulhout, M.; Vermoortele, F.; Horcajada, P.; Cunha, D.; Lee, J. S.; Chang, J.-S.; Gibson, E.; Daturi, M.; Lavalley, J.-C.; Vimont, A.; Beurroies, I.; De Vos, D. E., N/SHeterocyclic Contaminant Removal from Fuels by the Mesoporous Metal-Organic Framework MIL-100: The Role of the Metal Ion. Journal of the American Chemical Society 2013, 135 (26), 9849-9856.

8. Eftink, M. R.; Anusiem, A. C.; Biltonen, R. L., Enthalpyentropy compensation and heat capacity changes for protein-ligand interactions: general thermodynamic models and data for the binding of nucleotides to ribonuclease A. Biochemistry 2002, 22 (16), 38843896.

9. Lumry, R.; Rajender, S., Enthalpy-entropy compensation phenomena in water solutions of proteins and small molecules: a ubiquitous property of water. Biopolymers 1970, 9 (10), 1125-227.

10. Mills, E. A.; Plotkin, S. S., Protein Transfer Free Energy Obeys Entropy-Enthalpy Compensation. The Journal of Physical Chemistry B 2015, 119 (44), 14130-14144.

11. Pan, A.; Biswas, T.; Rakshit, A. K.; Moulik, S. P., EnthalpyEntropy Compensation (EEC) Effect: A Revisit. The Journal of Physical Chemistry B 2015, 119 (52), 15876-15884.
12. Pan, A.; Kar, T.; Rakshit, A. K.; Moulik, S. P., EnthalpyEntropy Compensation (EEC) Effect: Decisive Role of Free Energy. The Journal of Physical Chemistry B 2016, 120 (40), 10531-10539.

13. Brady, J.; Karplus, M., Configuration entropy of the alanine dipeptide in vacuum and in solution: a molecular dynamics study. Journal of the American Chemical Society 1985, 107 (21), 6103-6105.

14. Kollias, L.; Cantu, D. C.; Tubbs, M. A.; Rousseau, R.; Glezakou, V. A.; Salvalaglio, M., Molecular Level Understanding of the Free Energy Landscape in Early Stages of Metal-Organic Framework Nucleation. J Am Chem Soc 2019, 141 (14), 6073-6081.

15. Cantu, D. C.; McGrail, B. P.; Glezakou, V. A., Formation Mechanism of the Secondary Building Unit in a Chromium Terephthalate Metal-Organic Framework. Chemistry of Materials 2014, 26 (22), 6401-6409.

16. Taddei, M., When defects turn into virtues: The curious case of zirconium-based metal-organic frameworks. Coordination Chemistry Reviews 2017, 343, 1-24.

17. Howarth, A. J.; Liu, Y.; Li, P.; Li, Z.; Wang, T. C.; Hupp, J. T.; Farha, O. K., Chemical, thermal and mechanical stabilities of metal-organic frameworks. Nature Reviews Materials 2016, 1 (3).

18. Gutov, O. V.; Gonzalez Hevia, M.; Escudero-Adan, E. C.; Shafir, A., Metal-Organic Framework (MOF) Defects under Control: Insights into the Missing Linker Sites and Their Implication in the Reactivity of Zirconium-Based Frameworks. Inorg Chem 2015, 54 (17), 8396-400.

19. Howarth, A. J.; Peters, A. W.; Vermeulen, N. A.; Wang, T. C.; Hupp, J. T.; Farha, O. K., Best Practices for the Synthesis, Activation, and Characterization of Metal-Organic Frameworks. Chemistry of Materials 2016, 29 (1), 26-39.

20. Torres-Knoop, A.; Dubbeldam, D., Exploiting Large-Pore Metal-Organic Frameworks for Separations through Entropic Molecular Mechanisms. ChemPhysChem 2015, 16 (10), 2046-2067.

21. Sarkisov, L.; Martin, R. L.; Haranczyk, M.; Smit, B., On the Flexibility of Metal-Organic Frameworks. Journal of the American Chemical Society 2014, 136 (6), 2228-2231.

22. Balestra, S. R. G.; Bueno-Perez, R.; Hamad, S.; Dubbeldam, D.; Ruiz-Salvador, A. R.; Calero, S., Controlling Thermal Expansion: A Metal-Organic Frameworks Route. Chemistry of Materials 2016, 28 (22), 8296-8304

23. Torres-Knoop, A.; Poursaeidesfahani, A.; Vlugt, T. J. H.; Dubbeldam, D., Behavior of the Enthalpy of Adsorption in Nanoporous Materials Close to Saturation Conditions. Journal of Chemical Theory and Computation 2017, 13 (7), 3326-3339.

24. Gomez, D. A.; Sastre, G., From microscopic insights of H2 adsorption to uptake estimations in MOFs. Physical Chemistry Chemical Physics 2011, 13 (37), 16558.

25. Couck, S.; Rémy, T.; Baron, G. V.; Gascon, J.; Kapteijn, F.; Denayer, J. F. M., A pulse chromatographic study of the adsorption properties of the amino-MIL-53 (Al) metal-organic framework. Physical Chemistry Chemical Physics 2010, 12 (32), 9413.

26. Moosavi, S. M.; Boyd, P. G.; Sarkisov, L.; Smit, B., Improving the Mechanical Stability of Metal-Organic Frameworks Using Chemical Caryatids. ACS Cent Sci 2018, 4 (7), 832-839.

27. Lal, G.; Derakhshandeh, M.; Akhtar, F.; Spasyuk, D. M.; Lin, J.-B.; Trifkovic, M.; Shimizu, G. K. H., Mechanical Properties of a Metal-Organic Framework formed by Covalent Cross-Linking of Metal-Organic Polyhedra. Journal of the American Chemical Society 2018, 141 (2), 1045-1053.

28. Xie, X.-Y.; Wu, F.; Liu, X.; Tao, W.-Q.; Jiang, Y.; Liu, X.Q.; Sun, L.-B., Photopolymerization of metal-organic polyhedra: an efficient approach to improve the hydrostability, dispersity, and processability. Chemical Communications 2019.

29. Hess, B.; Bekker, H.; Berendsen, H. J. C.; Fraaije, J. G. E. M., LINCS: A linear constraint solver for molecular simulations. Journal of Computational Chemistry 1997, 18 (12), 1463-1472.

30. Darden, T.; York, D.; Pedersen, L., Particle Mesh Ewald an N.Log(N) Method for Ewald Sums in Large Systems. J. Chem. Phys. 1993, 98 (12), 10089-10092. 
31. Bussi, G.; Donadio, D.; Parrinello, M., Canonical sampling through velocity rescaling. $J$ Chem Phys 2007, 126 (1), 014101.

32. Berendsen, H. J. C.; Postma, J. P. M.; Vangunsteren, W. F.; Dinola, A.; Haak, J. R., Molecular-Dynamics with Coupling to an External Bath. Journal of Chemical Physics 1984, 81 (8), 3684-3690.

33. Jorgensen, W. L.; Chandrasekhar, J.; Madura, J. D.; Impey, R. W.; Klein, M. L., Comparison of Simple Potential Functions for Simulating Liquid Water. J. Chem. Phys. 1983, 79 (2), 926-935.

34. Jorgensen, W. L.; Tirado-Rives, J., The OPLS [optimized potentials for liquid simulations] potential functions for proteins, energy minimizations for crystals of cyclic peptides and crambin. $J \mathrm{Am}$ Chem Soc 1988, 110 (6), 1657-66.

35. Abraham, M. J.; Murtola, T.; Schulz, R.; Páll, S.; Smith, J. C.; Hess, B.; Lindahl, E., GROMACS: High performance molecular simulations through multi-level parallelism from laptops to supercomputers. Software $X$ 2015, 1-2, 19-25.

36. Tribello, G. A.; Bonomi, M.; Branduardi, D.; Camilloni, C.; Bussi, G., PLUMED 2: New feathers for an old bird. Computer Physics Communications 2014, 185 (2), 604-613.

37. Humphrey, W.; Dalke, A.; Schulten, K., VMD - Visual Molecular Dynamics. Journal of Molecular Graphics 1996, 14, 33-38.

38. Bonomi, M.; Bussi, G.; Camilloni, C.; Tribello, G. A.; Banáš, P.; Barducci, A.; Bernetti, M.; Bolhuis, P. G.; Bottaro, S.; Branduardi, D.; Capelli, R.; Carloni, P.; Ceriotti, M.; Cesari, A.; Chen, H.; Chen, W.; Colizzi, F.; De, S.; De La Pierre, M.; Donadio, D.; Drobot, V.; Ensing, B.; Ferguson, A. L.; Filizola, M.; Fraser, J. S.; Fu, H.; Gasparotto, P.; Gervasio, F. L.; Giberti, F.; Gil-Ley, A.; Giorgino, T.; Heller, G. T.; Hocky, G. M.; Iannuzzi, M.; Invernizzi, M.; Jelfs, K. E.; Jussupow, A.; Kirilin, E.; Laio, A.; Limongelli, V.; LindorffLarsen, K.; Löhr, T.; Marinelli, F.; Martin-Samos, L.; Masetti, M.; Meyer, R.; Michaelides, A.; Molteni, C.; Morishita, T.; Nava, M.; Paissoni, C.; Papaleo, E.; Parrinello, M.; Pfaendtner, J.; Piaggi, P.;
Piccini, G.; Pietropaolo, A.; Pietrucci, F.; Pipolo, S.; Provasi, D.; Quigley, D.; Raiteri, P.; Raniolo, S.; Rydzewski, J.; Salvalaglio, M.; Sosso, G. C.; Spiwok, V.; Šponer, J.; Swenson, D. W. H.; Tiwary, P.; Valsson, O.; Vendruscolo, M.; Voth, G. A.; White, A.; The, P. c., Promoting transparency and reproducibility in enhanced molecular simulations. Nature Methods 2019, 16 (8), 670-673.

39. Barducci, A.; Bussi, G.; Parrinello, M., Well-tempered metadynamics: a smoothly converging and tunable free-energy method. Physical Review Letters 2008, 100 (2), 020603.

40. Gimondi, I.; Tribello, G. A.; Salvalaglio, M., Building maps in collective variable space. J Chem Phys 2018, 149 (10), 104104.

41. Ryckaert, J.-P.; Bellemans, A., Molecular dynamics of liquid alkanes. Faraday Discussions of the Chemical Society 1978, 66, 95.

42. Tiwary, P.; Parrinello, M., A time-independent free energy estimator for metadynamics. $J$ Phys Chem B 2015, 119 (3), 736-42.

43. Caleman, C.; van Maaren, P. J.; Hong, M.; Hub, J. S.; Costa, L. T.; van der Spoel, D., Force Field Benchmark of Organic Liquids: Density, Enthalpy of Vaporization, Heat Capacities, Surface Tension, Isothermal Compressibility, Volumetric Expansion Coefficient, and Dielectric Constant. Journal of Chemical Theory and Computation 2012, 8 (1), 61-74.

44. van der Spoel, D.; van Maaren, P. J.; Caleman, C., GROMACS molecule \& liquid database. Bioinformatics 2012, 28 (5), 752-753.

45. Ferey, G.; Mellot-Draznieks, C.; Serre, C.; Millange, F.; Dutour, J.; Surble, S.; Margiolaki, I., A chromium terephthalate-based solid with unusually large pore volumes and surface area. Science 2005, 309 (5743), 2040-2.

46. Schauperl, M.; Podewitz, M.; Waldner, B. J.; Liedl, K. R., Enthalpic and Entropic Contributions to Hydrophobicity. Journal of Chemical Theory and Computation 2016, 12 (9), 4600-4610. 
Graphic entry for the Table of Contents (TOC)

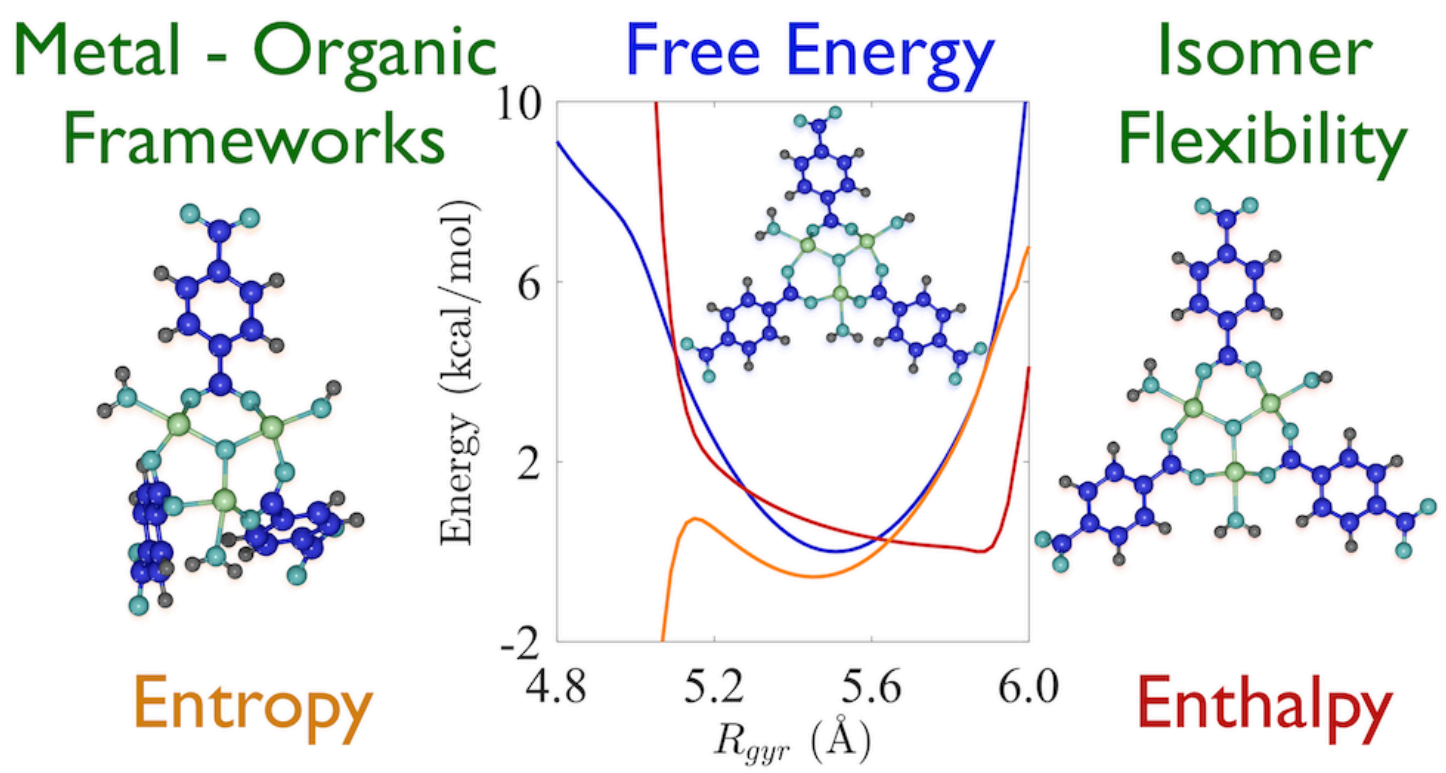

